\title{
Smart Distribution System by Using Binary Particle Swarm Optimization
}

\author{
Samuel Kefale Melese
}

Abstract - The electric Distribution System is subject to many disturbance which results power outage and material damage, and also due to its network topology power delivery to the customer is accompanied with the cost of power loss. Whenever reliability, power loss and resilience is an issue the distribution system must be designed to self healing. The power coming from the transmission line can be effectively distributed by managing automatically it's network configuration. This paper explores effective loss minimization for efficient smart electrical distribution power flow. The load on the distribution system is a continuously changing entity and hence a configuration which corresponds to a minimum loss at a particular load will not be so at a different load. Feeder reconfiguration is one of the techniques for loss minimization in a nonlinear network optimization problem which restructures the distribution feeders by changing the open/closed status of the sectionalizing and tie switches. and the solution for the problem involves a search over the possible configurations with multiple constraints. Binary particle swarm optimization method was applied to find the possible switching-options for loss reduction. Since distribution system is radial configuration backward forward load flow analysis is used. The algorithm is computationally efficient and determines the switching option that gives the maximum loss reduction in the system. For testing the effeteness of the method standard IEEE 33 bus system is used. The proposed algorithm is coded in MATLAB and power world simulator, to check the result obtained by MATLAB. The optimal configuration result shows that large amount of power loss was reduced from the initial configuration.

Index Terms-Binary Particle Swarm Optimization (BPSO), Feeder Reconfiguration, Power Loss, Backward Forward Load Flow.

\section{INTRODUCTION}

All the generated Electric power will not reach to the customer. There is certain amount dissipated or lost . Electric power loss can be defined as the difference between quantities of electricity produced and the amount recorded as sold to customers. Among the different techniques applied to optimize power loss feeder reconfiguration is one which uses the tie and sectionalize switch for distribution automation. Distribution Automation function provide a means to effectively manage the minute -by-minute continuous operation of distribution system. This involves collecting and analysing information from which to make decisions, implementing the appropriate decision and then verifying that the desired result is achieved. It is apparent that

Samuel Kefale Melese: Faculty of Electrical and Computer Engineering , Arba Minch University, Arba Minch, Ethiopia with the increasing complexity of power distribution systems, it is becoming essential to automate some tasks that have always been done manually [1]

Feeder reconfiguration involves study of switching options which enhances power loss, voltage stability under a given loading and generation condition. The improvement of distribution system efficiency is achieved only by altering topological structure of the power lines and does not involve any additional hardware like installation of SVC, capacitor bank, tap-changing transformers etc. The challenge in the proposed method however lies with the task of finding the optimum switching pattern that would maximize the overall voltage stability of the system and minimize the losses.

The electricity prices in deregulated markets are related to the system losses. This loss reduction can be done using effective and efficient computational tools which enhance power quality and reduce cost of energy at the consumer end [2]. In normal operating conditions, part of loads can be transferred from heavily loaded to relatively less heavily loaded feeders by network reconfiguration. Several methods have been proposed to solve the reconfiguration problem [3]

The load on the distribution system is a continuously changing entity and hence a configuration which corresponds to a minimum loss at a particular load will not be so at a different load. Hence there is a need to reconfigure feeders whenever there is a change in the load pattern on the system. A node encoding based on Prufer number is important for obtaining a radial structure in the distribution system. The Prufer number encoding can avoid the tedious "mesh check" algorithm. It can be proved that the prufer number encoding requires only (N-2) bits for encoding which is less than that required by the traditional branch encoding methods [4]. In most of the distribution systems in developing countries, feeders carry large currents to load points which lead to higher power loss (around 20\% ) resulting in poor power quality and higher electricity prices [5]

\section{FEEDER RECONFIGURATION}

The main objective of network reconfiguration is to find the network which is having economical losses during any 
desirable conditions exists in the network. Each configuration of the distribution system is defined by the status of switches

\section{Mathematical Model}

Given a distribution network with $\mathrm{n}$ nodes, the problem is a combinatorial non linear optimization. Combinatorial optimization problem involves selection of solution from among a finite, but very large of possible solution the Objective Function is Minimization of Real Power Losses

$$
\text { powerloss }=\sum_{\mathrm{i}=1}^{\mathrm{m}} \mathrm{I}_{\mathrm{i}}^{2} \cdot \mathrm{R}_{\mathrm{i}}
$$

Where :

- $I_{i}: \mathrm{i}^{\text {th }}$ branch current

- $\mathrm{R}_{\mathrm{i}}: \mathrm{i}^{\text {th }}$ branch resistance

Under the constraint of

- Keeping radiality which means no loops are allowed in the network,

- The loads must not be isolated without output supply from any feeder,

- $\quad$ Operating voltage at each node must be in safety range

$$
\mathrm{V}_{\mathrm{i}}^{\mathrm{min}} \leq \mathrm{V}_{\mathrm{i}} \leq \mathrm{V}_{\mathrm{i}}^{\max }
$$

- $\quad$ Power flow at each node must be kept in balance and power flow at each branch must be less than or equal its maximum capacity.

\section{2. $\quad$ System modeling}

The sampled distribution system used to test the proposed reconfiguration algorithm is Baran and $\mathrm{Wu}$ test system, which is shown in Figure 1. It consists of a feeder, 33 buses, 32 normally closed switches (sectionalizing switches), and 5 normally open switches (tie switches). The system is a three phase system, $12.6 \mathrm{KV}$. The system base loads are assumed constant and $\mathrm{S}_{\text {base }}$ is 100MVA.The maximum branch current is $250 \mathrm{~A}$ for all branches.

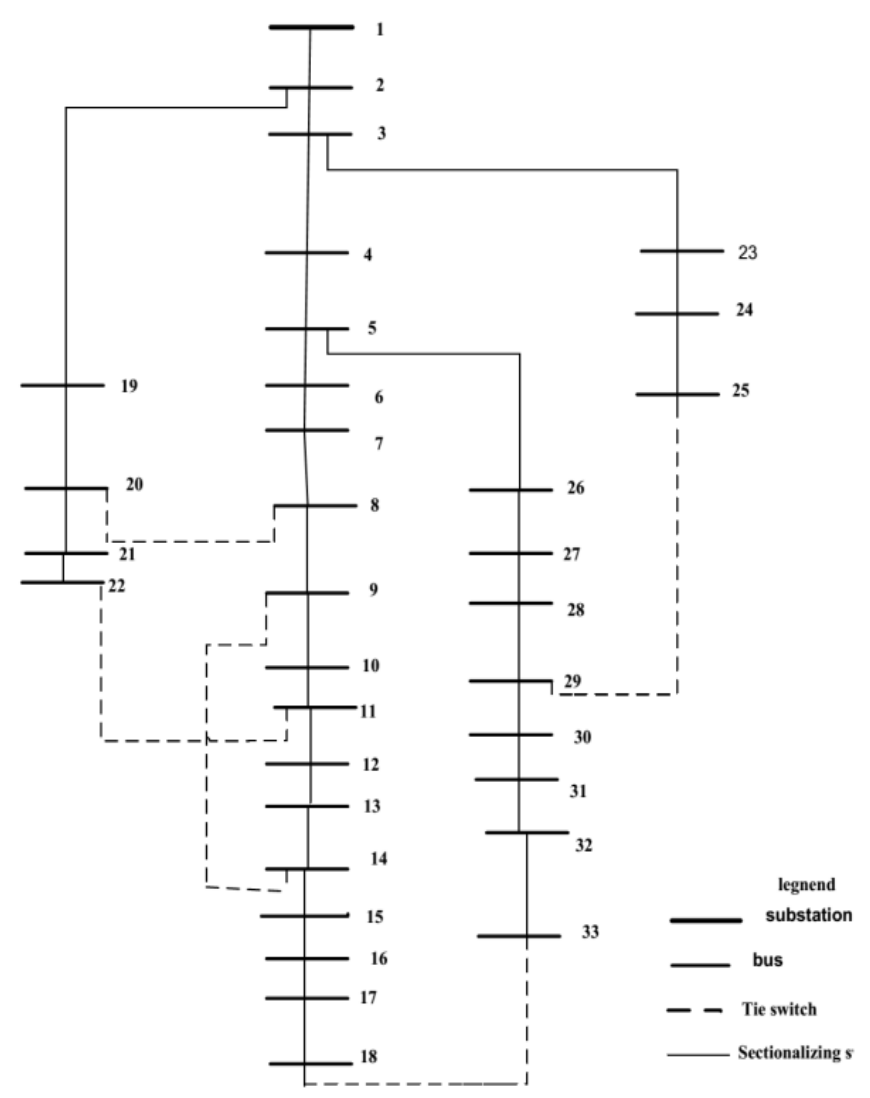

Fig.1: Initial configuration of the 33 bus distribution system

\section{BINARY PARTICLE SWARM OPTIMIZATION ALGORITHM}

Most distribution networks use sectionalizing-switches that are normally closed and tie-switches that are normally open and able to be optimized by using swarm intelligence. Binary particle swarm optimization is an evolutionary algorithm which has two model equations (velocity update and position update) in search of optimal solution [6].

$$
\begin{gathered}
V_{i}^{k+1}=\omega V_{i}^{k}+C_{1} * \text { rand }^{k}\left(\text { Pbest }_{i}-\right. \\
\left.X_{i}^{k}\right)+C_{2} * \text { rand } *\left(\text { gbest }_{i}-X_{i}^{k}\right) \\
\left.X_{i}^{k+1}=V_{i}^{k+1}+X_{i}^{k}\right)
\end{gathered}
$$

Where

- $: V_{i}^{k}$ is the $\mathrm{i}^{\text {th }}$ velocity component at iteration $\mathrm{k}$,

- rand: is random number between 0 and 1 ,

- $X_{i}^{k}$ : is the current position in the $\mathrm{i}^{\text {th }}$ dimension,

- $\mathrm{C} 1, \mathrm{C} 2$ : are the acceleration coefficients that are usually

set

to

2.0 , 
- : pbest $_{i}:$ is the personal best position in the $\mathrm{i}^{\text {th }}$ dimension,

- Gbest $t_{\mathrm{i}}$ : is the global best position in the $\mathrm{i}^{\text {th }}$ dimension and

- $\omega:$ is the inertia weight.

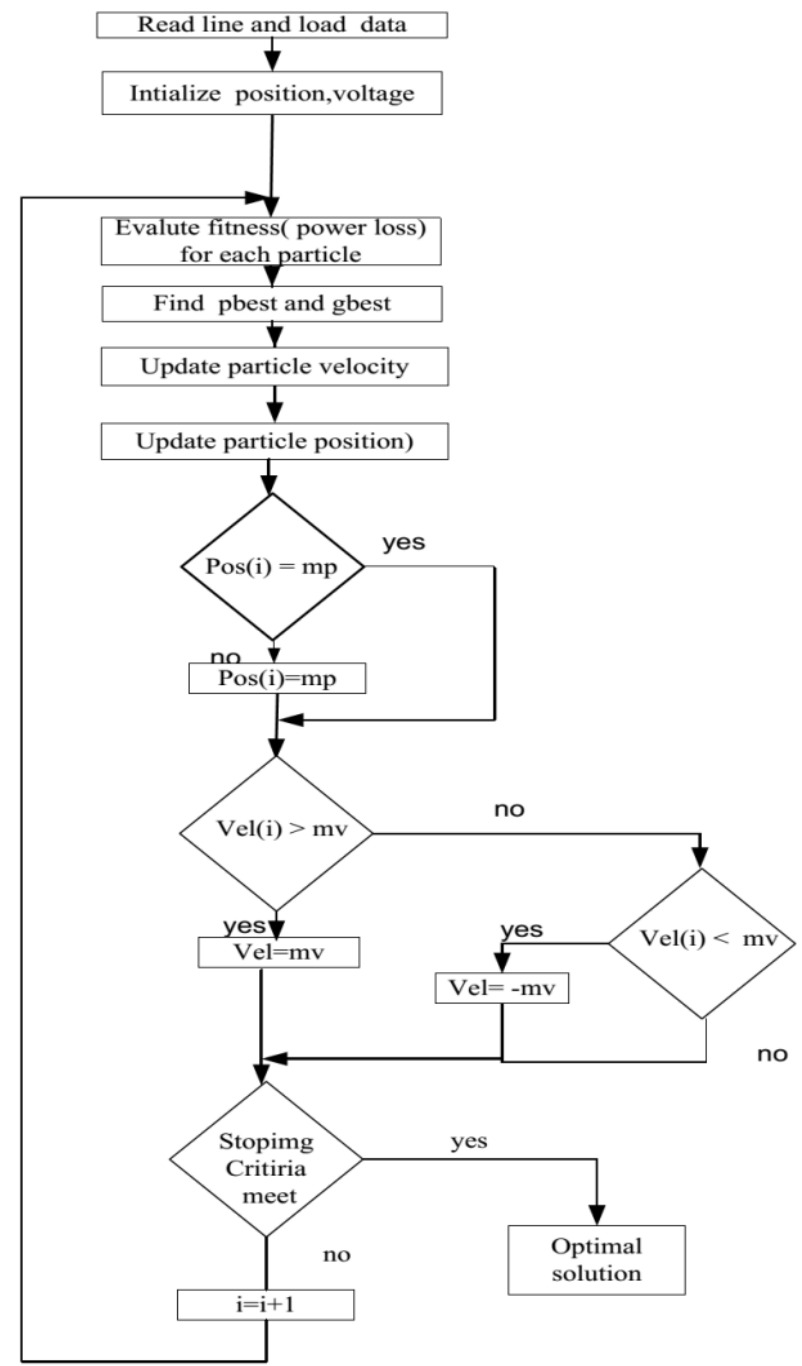

Fig. 2: BPSO algorithm for feeder reconfiguration

\section{RESULT AND DISCUSSION}

The result obtained by Appling BPSO on 33 buss system, the initial configuration of the system is changed into an optimal configuration of figure 3 as shown bellow and the optimal configuration is able to improve

- minimum voltage profile from 0.9131 P.u to 0.9378 ,

- power loss reduction from initial configuration i.e $202.6 \mathrm{KW}$ to $139,5 \mathrm{KW}$ which is $31.1 \%$ reduced from the initial loss . this result is better than Genetic Algorithm [3]

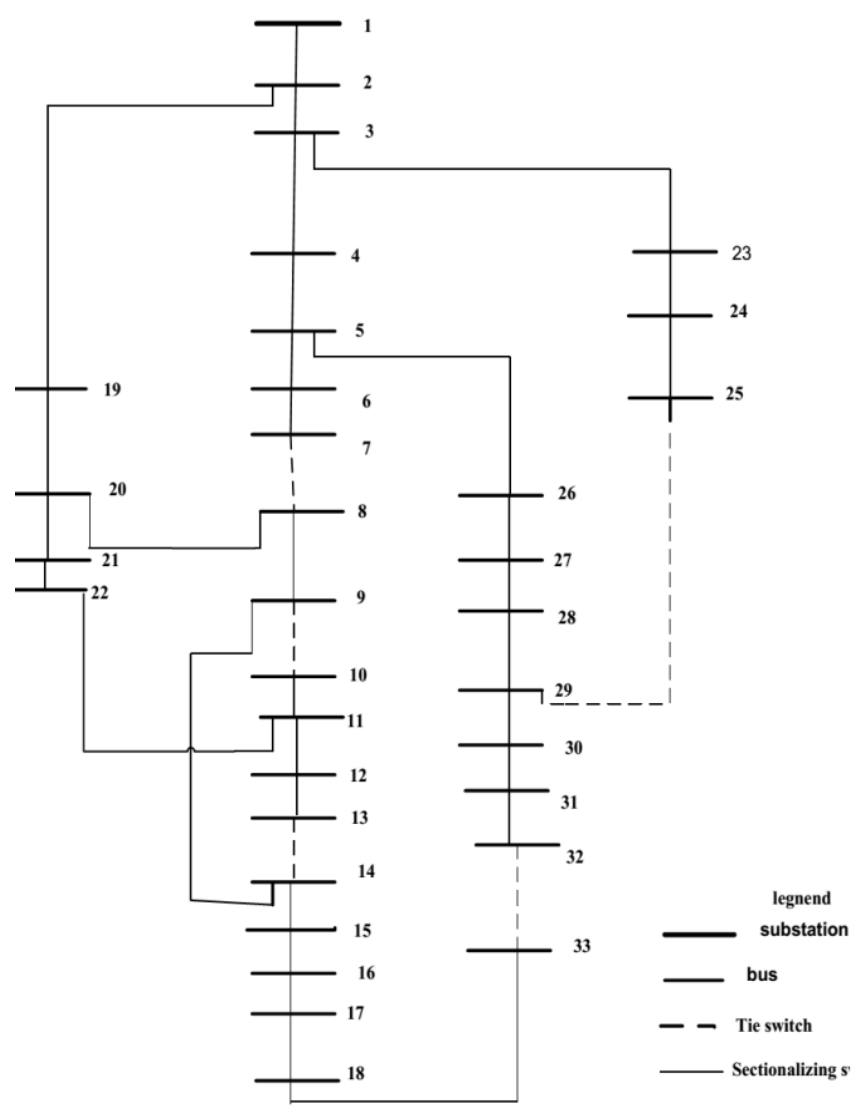

Fig. 3: optimal configuration

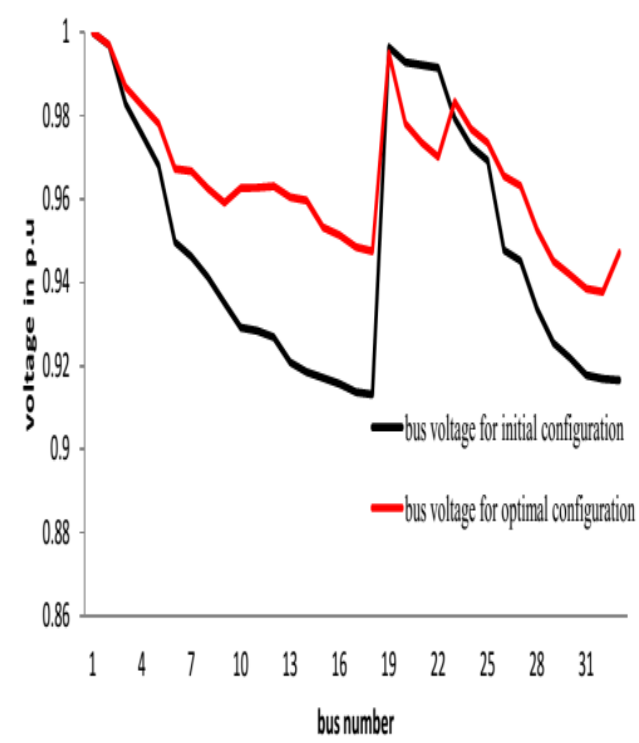

Fig. 4: voltage profile 


\section{CONCLUSION}

This paper provides a solution for highly nonlinear feeder reconfiguration problem with multi objective and multi constraints. BPSO algorithm is introduced with some modifications such as using an inertia weight that decreases linearly from 0.9 to 0.2 during the course of a simulation. This setting allows the BPSO to explore a large area at the start of the simulation. A modification in the number of iterations is also presented. The validity and effectiveness of the proposed BPSO algorithm is well proved by 33 buss systems. The result obtained shows that the method is superior to the existing methods in terms of power loss, computational efficiency and voltage profile improvement. Further more power loss reduction by using reconfiguration does not need any installation of capacitor placement, SVC, tap changing transformer or DG to reduce the system real power loss thus it is almost costless.

\section{ACKNOWLEDGMENT}

I would to thank my wife Lemlem Emwey and Dr. Vasireddy Raghuram for their support to purplish this paper.

\section{REFERENCE}

[1]. K.A Sambugari, "Power Loss Reduction in Radial Distribution System by Using Plant Growth Simulation Algorithm", Journal of Energy Technologies and Policy, Vol.2, No.3, 2012.

[2]. A. Alshehri, "Optimal reconfiguration of distribution networks using Ant colony method", King Saud University, 2007.

[3]. Y. Al-Mahroqi et al, "Reduction of Power Losses in Distribution Systems", world academy of science, engineering and Technology 63, 2012

[4]. K.A Sambugari, "Power Loss Reduction in Radial Distribution System by Using Plant Growth Simulation Algorithm", Journal of Energy Technologies and Policy, Vol.2, No.3, 2012.

[5]. S. Civanlar, et al., "Distribution feeder reconfiguration for loss reduction”, IEEE transactions' on Power Delivery, Vol. 3, No.3, 1988, pp. 1217-1223.

[6]. A. Y. Abdelaziz et al., "A Modified Particle Swarm Technique for Distribution Systems Reconfiguration", Department of Electrical Power and Machines, Faculty of Engineering, Ain Shams University, Cairo, Egypt.

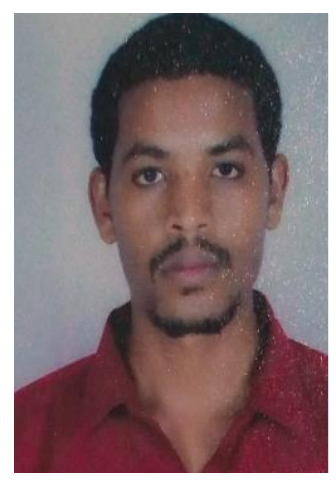

Samuel Kefale has obtained his M.Sc. Degree in Power System Engineering in 05 march 2014, from Bahir Dar University (Public) (Bahir Dar Institute of Technology) and B.Sc. Degree in Electrical and Computer Engineering, in 07 July 2010, from Bahir Dar University (Public) (Bahir Dar Institute of Technology. Presently, he is a Lecturer since 14 march 2014 and Chair of Electrical Power sytem Engineering since July 2018 in Arba Minch University Faculty of Electrical and Computer Engineering, Ethiopia.. Research area is Electrical Machine and Optimizations of Power system using Artificial intelligence. He published various reputed and scopus indexed journals in power systems and converters. 